\title{
Advances in imaging of AD biomarkers could aid early diagnosis
}

T he neuropathological effects of Alzheimer disease $(\mathrm{AD})$ can be clearly observed at autopsy, but identifying diagnostic and prognostic biomarkers in living people is less straightforward. In two recently published studies, new brain imaging protocols show promise for improving assessment of $\mathrm{AD}$.

A major hindrance for the early detection of $\mathrm{AD}$ has been the lack of biomarker specificity: though all patients with $\mathrm{AD}$ show neurodegeneration and elevated levels of amyloid- $\beta$ peptide, not all patients who develop these pathologies progress to $\mathrm{AD}$. In an article published online in Neurology, Clifford Jack and colleagues sought to elucidate the relationship between amyloid- $\beta$ deposition and neurodegeneration in older people.

Jack et al. recruited 252 cognitively normal people, aged 70 years or older, from the Mayo Clinic Study of Aging. The participants underwent a multimodal brain imaging examination: hippocampal volume was measured with MRI, and amyloid- $\beta$ levels were measured by PET using the ${ }^{11} \mathrm{C}-\mathrm{PiB}$ radiotracer.

More than half of the participants had no signs of amyloid build-up or hippocampal neurodegeneration. Approximately $22 \%$ of participants had increased amyloid- $\beta$ load with no signs of neurodegeneration, and $12 \%$ had reduced hippocampal volume but normal amyloid-PET results. Around $8 \%$ of patients demonstrated both types of pathology.

To measure changes in amyloid levels and atrophy in brain regions typically affected by $\mathrm{AD}$, the authors performed follow-up brain scans 1-6 years later. The annualized rate of amyloid accumulation was higher for participants showing increased amyloid load at baseline, whether or not they had also had significant hippocampal neurodegeneration, compared to the other groups. The rate of neurodegeneration was highest in the individuals who had both increased amyloid- $\beta$ deposition and hippocampal pathology at baseline, which suggests that this combination could indicate a preclinical form of $\mathrm{AD}$.

"Our data support the concept that the rate of amyloid- $\beta$ accumulation is biologically independent from neurodegeneration," says Jack, "but our data also suggest that amyloid- $\beta$ pathophysiology accelerates

neurodegeneration." These results support the model of $\mathrm{AD}$ development that posits three distinct stages of preclinical disease, which are characterized first by amyloid build-up, followed by neurodegeneration and cognitive impairment.

In future experiments, Jack and colleagues plan to measure tau protein levels by including an additional PET protocol with a tau-sensitive radiotracer. The development of such a PET ligand for use in patients with $\mathrm{AD}$ has recently been described in an article by Nobuyuki Okamura and colleagues published in Brain. "We have screened over 2000 compounds with high affinity for tau protein fibrils," says Okamura. The researchers have discovered two quinoline derivatives with particularly promising results in vitro.

Okamura et al. conducted PET scans with one of these novel radiotracers, ${ }^{18} \mathrm{~F}$-THK5105, in eight patients with clinically diagnosed $\mathrm{AD}$, and eight agematched controls. Structural MRI revealed that the patients with $\mathrm{AD}$ had reduced overall grey matter volume, particularly in the hippocampus. Patients with AD also had increased ${ }^{11} \mathrm{C}-\mathrm{PiB}$ binding compared to controls, indicating higher amyloid deposition.

Uptake of ${ }^{18} \mathrm{~F}$-THK5105 was also markedly increased in the patients with $\mathrm{AD}$, but the distribution of this radiotracer in the brain showed little overlap with the amyloid-PET results. Whereas ${ }^{11} \mathrm{C}-\mathrm{PiB}$ was mainly observed in areas of frontal and cingulate cortex, ${ }^{18} \mathrm{~F}$-THK5105 uptake was seen primarily in areas of the temporal lobe. This distribution pattern is similar to

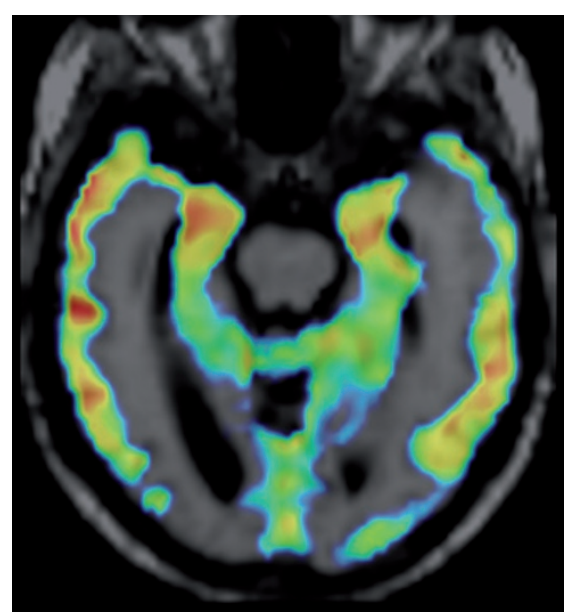

Distribution of ${ }^{18} \mathrm{~F}-\mathrm{THK} 5105$ in the brain of a patient with Alzheimer disease. Image courtesy of Nobuyuki Okamura.

the pattern of tau build-up typically seen in postmortem analyses of patients with $\mathrm{AD}$.

${ }^{18} \mathrm{~F}$-THK5105 uptake correlated with dementia severity-as assessed by the Mini Mental State Exam - and with levels of grey-matter degeneration. "These findings suggest that ${ }^{18} \mathrm{~F}$-THK5105 is useful for the noninvasive evaluation of tau pathology in humans, and could be employed to study longitudinal tau deposition in aging," concludes Okamura. A clinical trial of the second tau PET ligand-which might have favourable pharmacokinetics and binding selectivity-is in progress.

Together, these recent experiments highlight the potential for improving the diagnosis of $\mathrm{AD}$ through combined assessment of multiple biomarkers. Early and reliable detection of preclinical AD might improve treatment outcomes, and help to prevent the development of more serious impairment.

\section{Alex Chase}

\footnotetext{
Original articles Jack, C. R. et al. Rates of $\beta$-amyloid accumulation are independent of hippocampal neurodegeneration. Neurology doi:10.1212/ WNL.0000000000000386 | Okamura, N. et al. Non-invasive assessment of Alzheimer's disease neurofibrillary pathology using ${ }^{18}$ F-THK5105 PET. Brain doi:10.1093/brain/awu064
} 